\title{
Origins of Brown Dwarfs
}

\author{
Viki Joergens \\ Sterrewacht Leiden \\ PO Box 9513, 2300 RA Leiden, The Netherlands, \\ and \\ Max-Planck-Institut für Extraterrestrische Physik \\ Giessenbachstrasse 1, 85748 Garching, Germany \\ viki@strw. leidenuniv.nl
}

\begin{abstract}
The formation of objects below or close to the hydrogen burning limit is currently vividly discussed and is one of the main open issues in the field of the origins of stars and planets. Applying various observational techniques, we explored a sample of brown dwarfs and very low-mass stars in the ChaI star forming cloud at an age of only a few million years and determined fundamental parameters for their formation and early evolution.

Tracking the question of how frequent are brown dwarf binaries and if brown dwarfs have planets, one of the first radial velocity $(R V)$ surveys of brown dwarfs sensitive down to planetary masses is carried out based on high-resolution spectra taken with UVES at the VLT. The results hint at a low multiplicity fraction, which is in contrast to the situation for young low-mass stars.

Testing recent formation scenarios, which propose an ejection out of the birth place in the early accretion phase, we carried out a precise kinematic analysis of the brown dwarfs in our sample in comparison with $T$ Tauri stars in the same field. This yielded the first empirical upper limit for possible ejection velocities of a homogeneous group of brown dwarfs.

Rotation is a fundamental parameter for objects in this early evolutionary phase. By means of studying the line broadening of spectral features in the UVES spectra as well as by tracing rotational modulation of their lightcurves due to surface spots in photometric monitoring data, one of the first rotation rates of very young brown dwarfs have been determined.

In the light of the presented observational results, the current scenarios for the formation of brown dwarfs are discussed
\end{abstract}

\section{What are brown dwarfs ?}

Brown dwarfs fill the gap between low-mass stars and giant planets in the mass range of about 0.08 solar masses $\left(\mathrm{M}_{\odot}\right)$ and about 13 Jupiter masses $\left(\mathrm{M}_{\mathrm{Jup}}\right)^{1}$, depending on metallicity. They can never fully stabilize their luminosity by

\footnotetext{
${ }^{1} 1 \mathrm{M}_{\odot}=1047 \mathrm{M}_{\mathrm{Jup}}$
} 


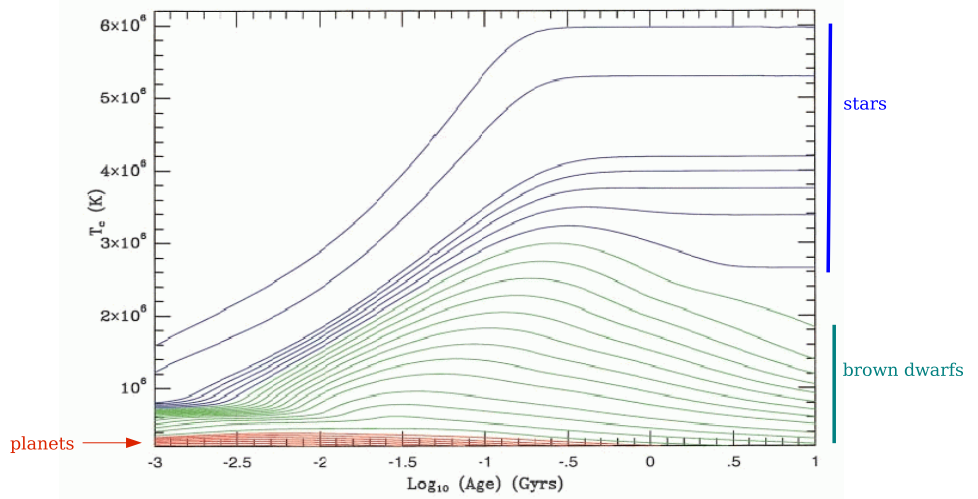

Figure 1: Evolution of the central temperature for stars, brown dwarfs and planets from $0.3 \mathrm{MJup}_{\mathrm{Ju}}(\sim$ Saturn$)$ to $0.2 \mathrm{M}_{\odot}\left(211 \mathrm{M}_{\mathrm{Jup}}\right)$ versus age. From Burrows et al. (2001). The upper seven curves, which end in the plot with constant central temperatures, represent stars, the 13 curves below represent brown dwarfs and the remaining curves at the smallest temperatures mark planets with masses $\leq 13 \mathrm{M}_{\text {Jup }}$. Note the increase (classical plasma), maximum and decrease (quantum plasma) of the central temperature for brown dwarfs.

hydrogen burning in contrast to stars and thus contract as they age until the electron gas in their interior is completely degenerate. At that point they have reached a final radius and become cooler and dimmer for their remaining life time. In contrast to planets brown dwarfs are able to fuse deuterium, which defines the lower mass limit of brown dwarfs. It is noted that although thermonuclear processes do not dominate the evolution of brown dwarfs, they do not only burn deuterium, but the more massive ones (masses above $\sim 0.065 \mathrm{M}_{\odot}$ ) burn lithium and may even burn hydrogen for a while. However, they do not burn hydrogen at a rate sufficient to fully compensate radiative losses.

Fig. 1 shows the evolution of the central temperature for low-mass stars (upper seven curves with constant central temperature at the end of the plot), brown dwarfs and planets (Burrows et al. 2001). The temperature for stars rises in the pre-main sequence phase due to the contraction on the Hayashi track until the interior is hot and dense enough to burn sufficient hydrogen to stabilize the central temperature and balance gravitational pressure on the hydrogen burning main sequence. For brown dwarfs, the central temperature also increases due to gravitational contraction in the first million to a few hun- 
dred million years. As the density in the interior increases part of the electron gas in the interior becomes degenerate. Electrons obey the Pauli exclusion principle, allowing one quantum state to be occupied by only one electron. For the compression of partly degenerate gas, energy is needed in order to bring the degenerate electrons closer. Therefore with onwardly contraction the temperature remains constant for a while and then starts to decrease. When the electron gas is completely degenerate, no further contraction is possible, the brown dwarf has reached its final radius and cools without compensation by compressional heating.

The existence of brown dwarfs was predicted by Kumar $(1962,1963)$ already in the early sixties. It followed a 30 year search for such faint objects until in 1995 almost at the same time three brown dwarfs were discovered independently: the Methane dwarf Gl $229 \mathrm{~B}$, a faint companion to a nearby M-dwarf, as cool as $1000 \mathrm{~K}$ (Nakajima et al. 1995, Oppenheimer et al. 1995) as well as two brown dwarfs in the Pleiades, Teide 1 (Rebolo et al. 1995) and PPl 15 (Stauffer et al. 1994). The latter object turned out to be in fact a pair of two gravitationally bound brown dwarfs (Basri \& Martín 1999). Up to now, more than 300 brown dwarfs have been detected in star forming regions (e.g. Béjar et al. 1999, Comerón et al. 2000), in young cluster (e.g. Pleiades, Martín et al. 2000) and in the field by the large infrared and optical surveys, DENIS (Delfosse et al. 1997), 2MASS (Kirkpatrick et al. 2000) and SLOAN (Hawley et al. 2002).

The year 1995 saw another spectacular discovery: the detection of the first extrasolar planet candidate with minimum mass around $\sim 1 \mathrm{M}_{\text {Jup }}$ orbiting the sun-like star 51 Peg (Mayor \& Queloz 1995). Since 1995, high-precision radial velocity (RV) surveys have detected more than 130 planets in orbit around stars (e.g. Mayor et al. 2003) with masses ranging from 14 Earth masses (e.g. Santos et al. 2004) to the brown dwarf border at about $13 \mathrm{M}_{\mathrm{Jup}}$.

Before 1995, no objects were known in the mass range between Jupiter in our own solar system and the lowest-mass stars. Today, the situation completely changed and this mass range is populated by hundreds of giant planets and brown dwarfs challenging our understanding of the formation of solar systems. Brown dwarfs play an important role in this discussion since they link the planet population to the stellar population.

\section{Brown dwarf formation models and predictions}

The substellar border defined by the hydrogen burning mass is a crucial dividing line with respect to the further evolution of an object but there is no obvious reason why it should be of any significance for the formation mechanism by which this objects was produced. Thus, by whichever process brown dwarfs are formed, it is expected to work continuously into the regime of very low-mass stars. Therefore, the term formation of brown dwarfs in this article stands for the formation of brown dwarfs and very low-mass stars even if not 
stated explicitly.

In the traditional picture of (low-mass) star and planet formation we assume that stars form by collapse and hierarchical fragmentation of molecular clouds (Shu et al. 1987), a process which involves the formation of a circumstellar disk due to conservation of angular momentum. The disk in turn is the birth place for planets, which form by condensation of dust and further growth by accretion of disk material. These ideas are substantially based on the situation in our own solar system. However, some properties of detected extrasolar planetary systems are difficult to explain within current theories of planet formation and it is under debate if our solar system is rather the exception than the rule. How brown dwarfs form is also an open issue but it is clear that a better knowledge of the mechanisms producing these transition objects between stars and planets will also clarify some open questions in the context of star and planet formation.

Brown dwarfs may be the high-mass extension of planets and form like giant planets in a disk around a star by either core accretion or disk instabilities (see Wuchterl et al. 2000 for a review). In the core accretion model, the formation of giant planets is initiated by the condensation of solids in a circumstellar disk, which accrete to larger bodies and form a rock/ice core. When this core reaches the critical mass for the accretion of a gas envelope $\left(\sim 10-15 \mathrm{M}_{\oplus}\right)$ a so called runaway gas accretion is triggered, which is only slowed down when the surrounding gas reservoir is depleted by the accreting protoplanet. If brown dwarfs are formed like giant planets, we should find them in orbit around their host star. However, brown dwarfs are found in large number as free-floating objects. Furthermore, in the ongoing highprecision RV surveys for extrasolar planets, an almost complete absence of brown dwarfs in close $(<3 \mathrm{AU})$, short-period orbits around solar-mass stars was found while these surveys detected more than 130 planets. This so-called 'brown dwarf desert' indicates that there is no continuity from planets to brown dwarfs in terms of formation.

Brown dwarfs may also form like stars by direct gravitational collapse and fragmentation of molecular clouds out of cloud cores, which are cold and dense enough to become Jeans-unstable for brown dwarf masses. Such small cloud cores have not yet been detected by radio observations but might have been missed due to insufficient detection sensitivity. However, on theoretical grounds, there is a so-called opacity limit for the fragmentation, i.e. a limiting mass, which can become Jeans-unstable: when reaching a certain density during the collapse the gas becomes optically thick and is heated up leading to an increase of the Jeans-mass (Low \& Lynden-Bell 1976). That might prevent the formation of (lower mass) brown dwarfs by direct collapse. Nevertheless, assuming brown dwarfs can form in that way, they would be the low-mass extension of the stellar population and should, therefore, have similar (scaled down) properties as young low-mass stars ( $\mathrm{T}$ Tauri stars). We know that $\mathrm{T}$ Tauri stars have a very high multiplicity fraction, in some star forming regions close to $100 \%$ (e.g. Leinert et al. 1993, Ghez et al. 1993, 1997, Köhler et al. 2000) indicating that the vast majority of low-mass stars is 
formed in binaries or higher order multiple systems. The stellar companions are thought to be formed by disk fragmentation or filament fragmentation in the circumstellar disk created by the collapse of the molecular cloud core. Therefore, we would also expect a high multiplicity fraction of brown dwarfs in this formation model. Furthermore, brown dwarfs should have circumstellar disks due to preservation of angular momentum during the collapse, harbour planets, and should have the same kinematical properties as the T Tauri stars in the same field.

Another idea is that brown dwarfs formed by direct collapse of unstable cloud cores of stellar masses and would have become stars if the accretion process was not stopped at an early stage by an external process before the object has accreted to stellar mass. It was proposed that such an external process can be the ejection of the protostar out of the dense gaseous environment due to dynamical interactions (Reipurth \& Clarke 2001). It is known that the dynamical evolution of gravitationally interacting systems of three or more bodies leads to frequent close two-body encounters and to the formation of close binary pairs out of the most massive objects in the system as well as to the ejection of the lighter bodies into extended orbits or out of the system with escape velocity (e.g. Valtonen \& Mikkola 1991). The escape of the lightest body is an expected outcome since the escape probability scales approximately as the inverse third power of the mass. Sterzik \& Durisen $(1995,1998)$ and Durisen et al. (2001) considered the formation of run-away $\mathrm{T}$ Tauri stars by such dynamical interactions of compact clusters.

The general expectations for the properties of brown dwarfs formed by the ejection scenario are a low binary frequency (maybe 5\%), no wide brown dwarf binaries and only close-in disks $(<5$-10 AU) since companions or disk material at larger separations will be truncated by the ejection process. Furthermore, the kinematics of ejected brown dwarfs might differ from non-ejected members of the cluster. Hydrodynamical calculations have shown that the collapse of a molecular cloud can produce brown dwarfs in this way (Bate et al. 2003, Delgado-Donate et al. 2003, Bate \& Bonnell 2005), while N-body simulations of the dynamical decay allowed the prediction of statistically significant properties of ejected brown dwarfs (Sterzik \& Durisen 2003, Delgado-Donate et al. 2004, Umbreit et al. 2005). However, the predictions differ significantly among the different models; this is further discussed in Sect.4.

An external process, which prevents the stellar embryo from further growth in mass can also be a strong UV wind from a nearby hot $\mathrm{O}$ or B star, which ionizes and photoevaporates the surrounding gas (Kroupa \& Bouvier 2003, Whitworth \& Zinnecker 2004). A significant disruption of the accretion envelope by photoevaporation will also lead generally to a low multiplicity fraction and limited disk masses. Since there is no such a hot star in the Cha I cloud, the brown dwarfs in this region cannot have been formed by this mechanism.

The various ideas for the formation of brown dwarfs need to be constrained by observations of brown dwarfs, key parameters are among others the multiplicity and kinematics, which have been studied for young brown dwarfs in ChaI as described in the following. Furthermore, rotation is an important 
parameter for the early evolution and it might reflect the interaction with a disk due to magnetic disk braking.

\section{Comprehensive observations of brown dwarfs and very low-mass stars in Cha I}

\subsection{Sample}

The observation of very young brown dwarfs and very low-mass stars allows insights into the formation and early evolution below or close to the substellar limit. One of the best grounds for such a study is the Cha I cloud, which is part of the larger Chamaeleon complex. At a distance of $160 \mathrm{pc}$, it is one of the closest sites of active low-mass star and brown dwarf formation. Comerón and coworkers initiated here one of the first surveys for young very low-mass objects down to the substellar regime by means of an $\mathrm{H} \alpha$ objective prism survey (Comerón et al. 1999, 2000; Neuhäuser \& Comerón 1998, 1999). They found twelve very low-mass M6-M8-type objects, Cha H $\alpha 1$ to 12, in the center of Cha I with ages of 1 to 5 Myrs, among which are four bona fide brown dwarfs and six brown dwarf candidates. Furthermore, they found or confirmed several very low-mass stars with masses smaller than $0.2 \mathrm{M}_{\odot}$ and spectral types M4.5-M5.5 in Cha I (B 34, CHXR 74, CHXR73 and CHXR 78C) as well as one 0.3 solar mass M2.5 star ( $\mathrm{Sz} 23$ ).

The membership to the Cha I cloud and therefore the youth of the objects is indicated by their $\mathrm{H} \alpha$ emission and has been confirmed by mediumresolution spectra, the detection of lithium absorption and consistent RVs (also by the here presented observations). The substellar nature is derived from the determination of bolometric luminosities and effective temperatures (converted by means of temperature scales from spectral types) and comparison with theoretical evolutionary tracks in the Hertzsprung-Russell diagram (HRD).

Another substellar test applicable to young brown dwarfs was developed from a test for old brown dwarfs based on lithium absorption (Rebolo et al. 1992) to the age-independent statement, that any object with spectral type Mr or later that shows lithium is substellar (Basri 2000). Since all $\mathrm{Cha} \mathrm{H} \alpha$ objects show lithium absorption in their spectra (Comerón et al. 2000, Joergens \& Guenther 2001), the four objects Cha H $\alpha$ 1, 7, 10 and 11 (M7.5-M8) are thus bona fide brown dwarfs, with masses in the range of about $0.03 \mathrm{M}_{\odot}$ to $0.05 \mathrm{M}_{\odot}$, Cha H $\alpha 2,3,6,8,9,12$ (M6.5-M7) can be classified as brown dwarf candidates and $\mathrm{ChaH} \alpha 4$ and 5 (M6) are most likely very low-mass stars with $0.1 \mathrm{M}_{\odot}$. An error of one subclass in the determination of the spectral type was taken into account.

We observed most of these ten brown dwarfs and brown dwarf candidates and seven (very) low-mass stars by means of high-resolution spectroscopy with UVES at the $8.2 \mathrm{~m}$ telescope at the VLT and by means of a photometric monitoring campaign at a $1.5 \mathrm{~m}$ telescope. 


\subsection{High-resolution UVES spectroscopy}

High-resolution spectra have been taken for the brown dwarfs and low-mass stars Cha $\mathrm{H} \alpha 1-8$ and $\mathrm{Cha \textrm {H }} \alpha 12$, B34, CHXR 74 and Sz 23 between the years 2000 and 2004 with the cross-dispersed UV-Visual Echelle Spectrograph (UVES, Dekker et al. 2000) attached to the $8.2 \mathrm{~m}$ Kueyen telescope of the Very Large Telescope (VLT) operated by the European Southern Observatory at Paranal, Chile. The wavelength regime from $6600 \AA$ to $10400 \AA$ was covered with a spectral resolution of $\lambda / \Delta \lambda=40000$. For each object at least two spectra separated by a few weeks have been obtained in order to monitor time dependence of the RVs. For several objects, more than two and up to twelve spectra have been taken.

After standard reduction, we have measured RVs by means of cross-correlating plenty of stellar lines of the object spectra with a template spectrum. In order to achieve a high wavelength and therefore RV precision, telluric $\mathrm{O}_{2}$ lines have been used as wavelength reference. A RV precision between $40 \mathrm{~m} \mathrm{~s}^{-1}$ and $670 \mathrm{~m} \mathrm{~s}^{-1}$, depending on the $\mathrm{S} / \mathrm{N}$ of the individual spectra, was achieved for the relative RVs. The errors are based on the standard deviation of two consecutive single spectra. An additional error of about $300 \mathrm{~m} \mathrm{~s}^{-1}$ has to be taken into account for the absolute RVs due to uncertainties in the zero point of the template. These RVs are one of the most precise ones for young brown dwarfs and very low-mass stars available up to now.

Based on the time-resolved RVs, a RV survey for planetary and brown dwarf companions to the targets was carried out (Sect. 国). The mean RVs were explored in a kinematic study of this group of brown dwarfs in Sect.4. Additionally, projected rotational velocities $v \sin i$ were derived from linebroadening of spectral features (Sect.6), lithium equivalent width has been measured to confirm youth and membership to the Cha I star forming region and the CaII IR-triplet has been studied as an indicator for chromospheric activity. Results have been or will be published in Joergens \& Guenther (2001), Joergens (2003), Joergens et al. (2005a,b).

\subsection{Photometric monitoring}

In order to study the time-dependent photometric behaviour of the brown dwarfs and very low-mass stars, we monitored a $13^{\prime} \times 13^{\prime}$ region in the Cha I cloud photometrically in the Bessel $\mathrm{R}$ and the Gunn i filter in six consecutive half nights with the CCD camera DFOSC at the Danish $1.5 \mathrm{~m}$ telescope at La Silla, Chile in May and June 2000. Differential magnitudes were determined relative to a set of reference stars in the same field by means of aperture photometry. The field contained all of the targets introduced in the previous sections, however, CHXR 74 and Sz 23 were too bright and have been saturated in the images, while $\mathrm{Cha \textrm {H }} \alpha 1,7,9,10$ and 11 were too faint for the size of the telescope.

Based on the lightcurves for Cha $\mathrm{H} \alpha 2,3,4,5,6,8,12$, B 34, CHXR 78C and CHXR 73, we searched for periodic variabilities and were able to deter- 
mine absolute rotation periods for several brown dwarfs and very low-mass stars based on brightness modulations due to surface activity (Sect.6] Joergens et al. 2003b).

\section{Kinematics of brown dwarfs in Cha I and the ejection scenario}

It was proposed that brown dwarfs might form in a star-like manner but have been prevented from accreting to stellar masses by the early ejection out of their birth place (Sect.21). The ejection process might have left an observable imprint in the kinematics of ejected members of a cluster in comparison to that of non-ejected members. Therefore, we are studying the kinematics of our target brown dwarfs in ChaI by means of precise mean RVs measured from UVES spectra. We find that the seven brown dwarfs and brown dwarf candidates and two $0.1 \mathrm{M}_{\odot}$ stars in Cha I (spectral types M6-M8) differ very less from each other in terms of their RV. The sample has a mean RV of $15.7 \mathrm{~km} \mathrm{~s}^{-1}$ and a RV dispersion measured in terms of standard deviation of only $0.9 \mathrm{~km} \mathrm{~s}^{-1}$. The total covered RV range is $2.6 \mathrm{~km} \mathrm{~s}^{-1}$. We note, that in previous publications (Joergens \& Guenther 2001, Joergens 2003), the dispersion was measured in terms of full width at half maximum (fwhm) of a Gaussian distribution (which is related to the standard deviation $\sigma$ of the Gaussian by fwhm $=\sigma \sqrt{8 \ln 2}$ ) following the procedure of radio astronomers. Based on new RV data and an improved data analysis, the here presented kinematic study (Joergens et al. 2005b) is a revised version of the previous ones. The new fwhm for the brown dwarfs is $2.1 \mathrm{~km} \mathrm{~s}^{-1}$, which is consistent with the previous value of $2.0 \mathrm{~km} \mathrm{~s}^{-1}$.

In order to compare the results for the brown dwarfs in Cha I with the RV distribution of higher-mass stellar objects in this cluster, we compiled all $\mathrm{T}$ Tauri stars confined to the same region for which RVs have been measured with a precision of $2 \mathrm{~km} \mathrm{~s}^{-1}$ or better from the literature (Walter 1992, Dubath et al. 1996, Covino et al. 1997, Neuhäuser \& Comerón 1999), from Guenther et al. (in prep., see Joergens \& Guenther 2001) and from our own measurements based on UVES spectra for M-type T Tauri stars. The compiled 25 T Tauri stars (spectral types M5-G2) have a mean RV of $14.7 \mathrm{~km} \mathrm{~s}^{-1}$ and a RV dispersion in terms of standard deviation of $1.3 \mathrm{~km} \mathrm{~s}^{-1}$. The dispersion given in terms of fwhm is $3.1 \mathrm{~km} \mathrm{~s}^{-1}$. Compared to the previous studies (Joergens \& Guenther 2001, Joergens 2003), the T Tauri sample and RVs have been also revised by taking into account new UVES-based RVs obtained by us in 2002 and 2004 as well as overlooked RV measurements by Walter (1992). Furthermore, based on a recent census of Cha I (Luhman 2004) an up-to-date check of membership status was possible. The new standard deviation of $1.3 \mathrm{~km} \mathrm{~s}^{-1}$ differs only slightly from the previous one of $1.5 \mathrm{~km} \mathrm{~s}^{-1}$.

The resulting RV distributions of the T Tauri stars and the brown dwarfs are displayed in Fig. 22 in form of a histogram. The mean RV of the M6-M8 type brown dwarfs and very low-mass stars $\left(15.7 \mathrm{~km} \mathrm{~s}^{-1}\right)$ is consistent within 


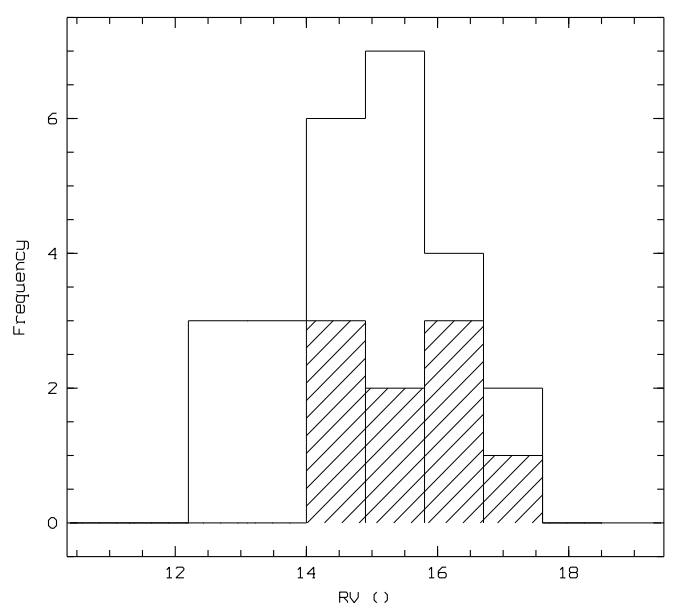

Figure 2: Distribution of mean RVs in $\mathrm{km} \mathrm{s}^{-1}$ for nine brown dwarfs and very low-mass stars with masses $\leq 0.1 \mathrm{M}_{\odot}$ (hashed) and for $25 \mathrm{~T}$ Tauri stars in Cha I.

the errors with that of the T Tauri stars in the same field $\left(14.7 \mathrm{~km} \mathrm{~s}^{-1}\right)$ and with that of the molecular gas of the surrounding $\left(15.3 \mathrm{~km} \mathrm{~s}^{-1}\right.$, Mizuno et al. 1999). This confirms the membership of the substellar population to the Cha I star forming cloud.

Furthermore, we find that the RV dispersion of the brown dwarfs $\left(0.9 \mathrm{~km} \mathrm{~s}^{-1}\right)$ is slightly smaller than that of the T Tauri stars in the same field $\left(1.3 \mathrm{~km} \mathrm{~s}^{-1}\right)$ and slightly larger than that of the surrounding molecular gas (the fwhm of $1.2 \mathrm{~km} \mathrm{~s}^{-1}$ given by Mizuno et al. (1999) translates to a standard deviation of $0.5 \mathrm{~km} \mathrm{~s}^{-1}$ using the above given formula). For the comparison of the kinematic properties of brown dwarfs and stellar objects we have set the dividing line between the two populations arbitrarily at $0.1 \mathrm{M}_{\odot}$ knowing that this is not exactly the substellar border. However, the process forming brown dwarfs is expected to work continuously into the regime of very low-mass stars and it is a priori unknown if and for which mass range a different formation mechanism is operating. In a more detailed study (Joergens et al 2005b), we calculate the dispersion also for different choices of this dividing line but see no significant differences in the results.

RVs are tracing only space motions in the direction of the line-of-sight and the studied brown dwarfs could have a larger three-dimensional velocity dispersion. However, the studied brown dwarfs in Cha I have an age of 15 Myr and occupy a field of less than $12^{\prime} \times 12^{\prime}$ at a distance of $160 \mathrm{pc}$. Brown dwarfs born within this field and ejected during the early accretion phase in directions with a significant fraction perpendicular to the line-of-sight, would have flown out of the field a long time ago for velocities of $0.5 \mathrm{~km} \mathrm{~s}^{-1}$ or larger (Joergens et al. 2003a). Therefore, the measurement of RVs is a suitable 
method to test if objects born and still residing in this field have significantly high velocities due to dynamical interactions during their formation process.

Numerical simulations of brown dwarf formation by the ejection scenario differ in their predictions of the resulting velocities over a wide range. In general, the ejection velocity scales with the inverse of the square root of the distance of the closest approach in the encounter that led to the ejection (Armitage \& Clarke 1997). The resulting velocity distribution depends also on the gravitational potential of the cluster since it defines if an ejected object with a certain velocity can escape or is ejected only in a wide eccentric orbit (e.g. Kroupa \& Bouvier 2003). Furthermore, the kinematic signature of the ejection can be washed out if only part of the brown dwarfs in a cluster are formed according to the ejection scenario while a significant fraction is formed by other mechanisms (disk instabilities alone or direct collapse above the fragmentation limit), or when, on the other hand, all (sub)stellar objects in a cluster undergo significant dynamical interactions.

Hydrodynamic calculations by Bate et al. (2003) and Bate \& Bonnell (2005) predict velocity dispersions of $2.1 \mathrm{~km} \mathrm{~s}^{-1}$ and $4.3 \mathrm{~km} \mathrm{~s}^{-1}$, resp., and no kinematic difference between brown dwarfs and T Tauri stars. These calculations are performed for much denser star forming regions than Cha I and an extrapolation of their results to the low-density Cha I cloud might lead to a consistency with our observed RV dispersion of $0.9 \mathrm{~km} \mathrm{~s}^{-1}$ for brown dwarfs and only slightly larger for T Tauri stars. However, comparison with Delgado-Donate et al. (2004) indicates that the dependence of the velocity dispersion on the stellar density is not yet well established. N-body simulations by Sterzik \& Durisen (2003) predict that $25 \%$ of brown dwarf singles have a velocity smaller than $1 \mathrm{~km} \mathrm{~s}^{-1}$, that is much lower than our observations of $67 \%$ of brown dwarfs in Cha I having RVs smaller than $1 \mathrm{~km} \mathrm{~s}^{-1}$. Also the high velocity tail found by the authors of $40 \%$ single brown dwarfs having higher velocities than $1.4 \mathrm{~km} \mathrm{~s}^{-1}$ and $10 \%>5 \mathrm{~km} \mathrm{~s}^{-1}$ is not seen in our data, were none has a RV deviating by the mean by more than $1.4 \mathrm{~km} \mathrm{~s}^{-1}$. Recent N-body calculations by Umbreit et al. (2005) showed that the ejection velocities depend strongly on accretion and by assuming different accretion models and rates, the authors predict an even more pronounced high-velocity tail with $60 \%$ to $80 \%$ single brown dwarfs having velocities larger than $1 \mathrm{~km} \mathrm{~s}^{-1}$. This is also much larger than found by our observations, where only about $30 \%$ have velocities $>1 \mathrm{~km} \mathrm{~s}^{-1}$. One might argue that the non-detection of a high velocity tail in our data can be attributed to the relatively small size of our sample, on the other hand, the current N-body simulations do not take into account the gravitational potential of the cluster, which might cause a suppression of the highest velocities.

To conclude, the observed values of our kinematic study provide the first observational constraints for the velocity distribution of a group of very young brown dwarfs and show that they have a RV dispersion of $0.9 \mathrm{~km} \mathrm{~s}^{-1}$, no high-velocity tail and their RVs are not more dispersed than that of T Tauri stars in the same field. These observed velocities are smaller than any of the theoretical predictions for brown dwarfs formed by the ejection scenario, 
which might be attributed to the lower densities in Cha I compared to some model assumptions, to shortcomings in the models, like neglection of feedback processes (Bate et al. 2003, Bate \& Bonnell 2005, Delgado-Donate et al. 2004) or of the cluster potential (Sterzik \& Durisen 2003, Umbreit et al. 2005), or to the fact that our sample is statistically relatively small. The current conclusion is that either the brown dwarfs in Cha I have been formed by ejection but with smaller velocities as theoretically predicted or they have not been formed in that way.

\section{$5 \quad$ RV Survey for planets and brown dwarf companions with UVES}

Based on the precise RV measurements in time resolved UVES spectra (Sect.3.2), we are carrying out a RV survey for (planetary and brown dwarf) companions to the young brown dwarfs and very low-mass stars in the Cha I cloud.

The detection of planets around brown dwarfs as well as the detection of young spectroscopic brown dwarf binaries would be an important clue towards the formation of brown dwarfs. So far, no planet is known orbiting a brown dwarf (the recent publication of a direct image of a candidate for a $5 \mathrm{M}_{\mathrm{Jup}}$ planet around a $25 \mathrm{M}_{\mathrm{Jup}}$ brown dwarf by Chauvin et al. (2004) is very exciting but still very tentative since it might very well be a background object). There have been detected several brown dwarf binaries, among them there are three spectroscopic, and hence close systems (Basri \& Martín 1999, Guenther \& Wuchterl 2003). However, all known brown dwarf binaries are fairly old and it is not yet established if the typical outcome of the brown dwarf formation process is a binary or multiple brown dwarf system or a single brown dwarf.

Furthermore, the search for planets around very young as well as around very low-mass stars and brown dwarfs is interesting since the detection of young planets as well as a census of planets around stars of all spectral types, and maybe even around brown dwarfs, is an important step towards the understanding of planet formation. It would provide empirical constraints for planet formation time scales. Furthermore, it would show if planets can exist around objects which are of considerably lower mass and surface temperature than our sun.

\subsection{RV constant objects}

The RVs for the brown dwarfs and very low-mass stars Cha H $\alpha 1,2,3,4,5$, $6,7,12$ and B 34 are constant within the measurements uncertainties of $2 \sigma$ for Cha $\mathrm{H} \alpha 4$ and of $1 \sigma$ for all others, as displayed in Figs. 3. 4 From the non-detections of variability, we have estimated upper limits for the projected masses $M_{2} \sin i$ of hypothetical companions for each object ${ }^{2}$. They range

\footnotetext{
${ }^{2}$ Spectroscopic detections of companions allow in general no absolute mass determination but only the derivation of a lower limit of the companion mass $M_{2} \sin i$ due to the unknown inclination $i$.
} 

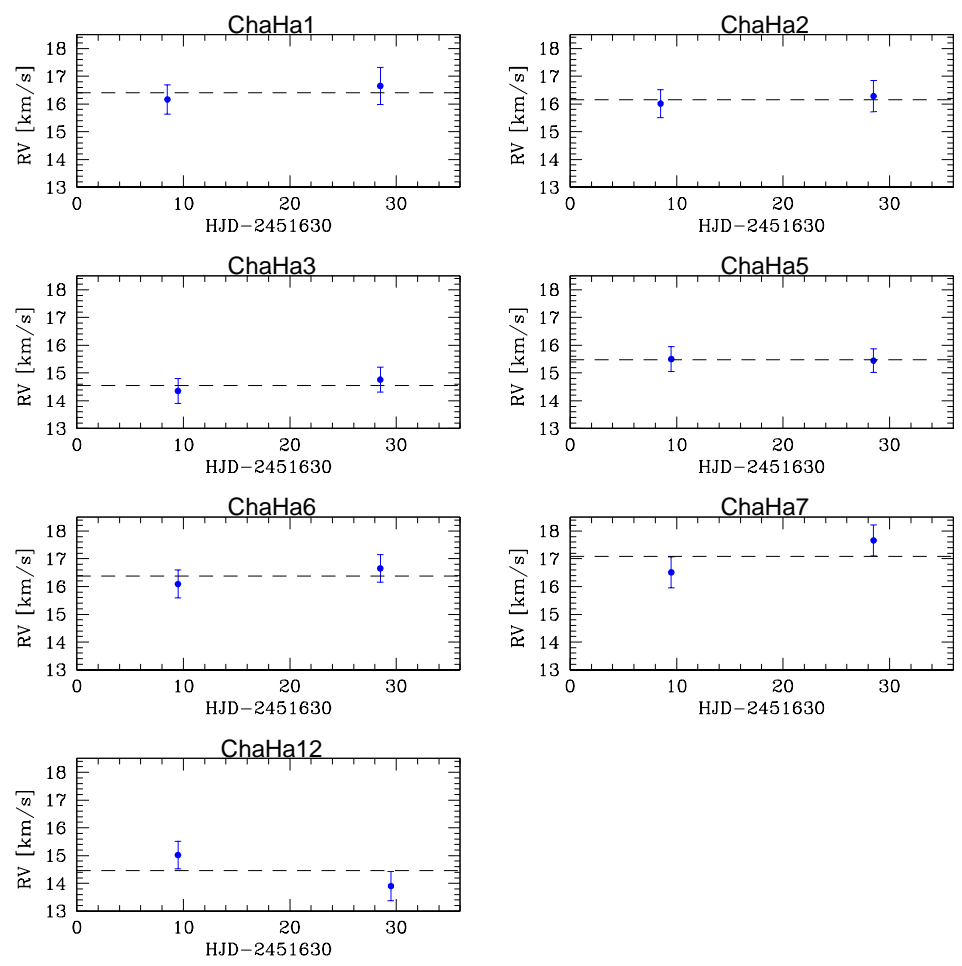

Figure 3: RV constant objects: RV vs. time in Julian days for brown dwarfs and very low-mass stars (M6-M8) in Cha I based on high-resolution UVES/VLT spectra. Error bars indicate $1 \sigma$ errors.

between $0.1 \mathrm{M}_{\mathrm{Jup}}$ and $1.5 \mathrm{M}_{\mathrm{Jup}}$ assuming a circular orbit, a separation of $0.1 \mathrm{AU}$ between companion and primary object and adopting primary masses from Comerón et al. $(1999,2000)$. The used orbital separation of $0.1 \mathrm{AU}$ corresponds to orbital periods ranging between 30 and 70 days for the masses of these brown dwarfs and very low-mass stars.

That means, that these nine brown dwarfs and very low-mass stars with spectral types M5-M8 and masses $\leq 0.12 \mathrm{M}_{\odot}$ show no RV variability down to Jupitermass planets. There is, of course, the possibility that present companions have not been detected due to non-observations at the critical orbital phases. Furthermore, long-period companions may have been missed since for all of them but $\mathrm{Cha} \mathrm{H} \alpha 4$, the time base of the observations does not exceed two months. 

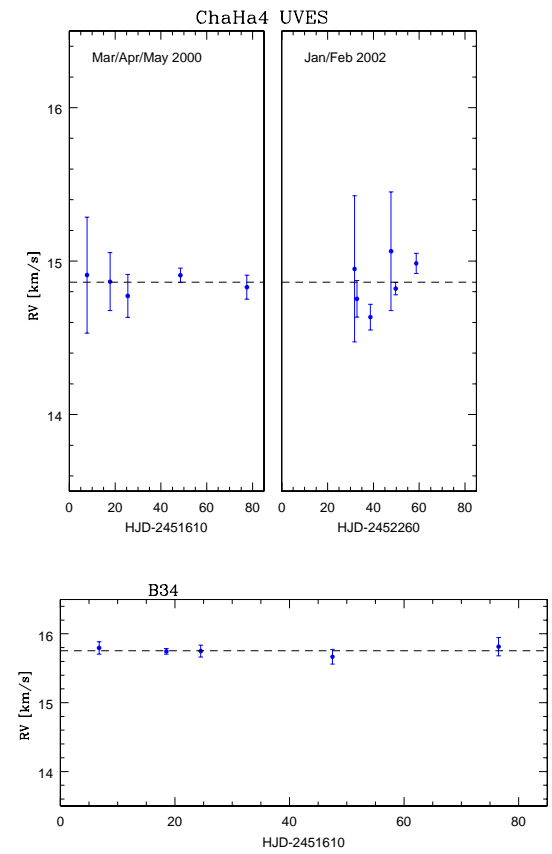

Figure 4: RV constant objects (continued): RV vs. time in Julian days for very low-mass stars $\left(\sim 0.1 \mathrm{M}_{\odot}\right)$ in Cha I based on high-resolution UVES/VLT spectra. Error bars indicate $1 \sigma$ errors.

\section{$5.2 \quad \mathrm{RV}$ variable objects}

For three objects, we have found significant RV variations, namely for the brown dwarf Cha $\mathrm{H} \alpha 8$ and the low-mass stars CHXR $74\left(\sim 0.17 \mathrm{M}_{\odot}\right)$ and $\mathrm{Sz} 23$ $\left(\sim 0.3 \mathrm{M}_{\odot}\right)$ as shown in Fig. [5. The variability characteristic differs among the three objects. Sz 23 shows variability on time scales of days with no difference in the mean values of RVs recorded in 2000 and in 2004. On the other hand, $\mathrm{Cha} \mathrm{H} \alpha 8$ and $\mathrm{CHXR} 74$ show only very small amplitude variations or no variations at all on time scales of days to weeks, whereas the mean RV measured in 2000 differs significantly from the one measured years later, namely in 2002 for Cha $\mathrm{H} \alpha 8$ and in 2004 for CHXR 74, respectively, hinting at variability periods of the order of months or longer.

One possibility for the nature of these RV variations is that they are the Dopplershift caused by the gravitational force of orbiting companions. The poor sampling does not allow us to determine periods of the variations but based on the data we suggest that the period for Cha $\mathrm{H} \alpha 8$ is 150 days or longer. A $150 \mathrm{~d}$ period would corresponds to a $6 \mathrm{M}_{\mathrm{Jup}}$ planet orbiting at a separation of $0.2 \mathrm{AU}$ around Cha8. For longer periods the orbital separations as well as the mass of the companion would be larger. For CHXR74, a period 
of about 200 days would be able to explain the RV data of 2000 and 2004 corresponds to a $15 \mathrm{M}_{\text {Jup }}$ brown dwarf orbiting CHXR74 at a separation of $0.4 \mathrm{AU}$.

The other possibility is that they are caused by surface activity since prominent surface spots can cause a shifting of the photo center at the rotation period (see also Sect.6.2). The upper limits for the rotational periods of Cha $\mathrm{H} \alpha$ 8, CHXR 74 and $\mathrm{Sz} 23$ are $1.9 \mathrm{~d}, 4.9 \mathrm{~d}$ and $2.1 \mathrm{~d}$, respectively, based on projected rotational velocities $v \sin i$ (Sect.6. Joergens \& Guenther 2001). Thus, the time-scale of the RV variability of Sz 23 is of the order of the rotation period and could be a rotation-induced phenomenon. In contrast to the other RV variable objects, Sz 23 is also displaying significant emission in the CaII IR triplet lines, which is an indicator for chromospheric activity. A further study of time variations of these lines is underway.

The RV variability of Cha $\mathrm{H} \alpha 8$ and CHXR 74 on time scales of months to years cannot be explained by being rotational modulation. If caused by orbiting companions, the detected RV variations of CHXR 74 and $\mathrm{Cha} \mathrm{H} \alpha 8$ correspond to giant planets of a few Jupiter masses with periods of several months.

\subsection{Discussion: Multiplicity, separations, RV noise}

Fig. [6] displays for all targets of the RV survey the measured RV semiamplitude (for RV constant objects the upper limit for it) versus their mass, as adopted from Comerón et al. (1999, 2000). The three RV variable objects have RV amplitudes above $1 \mathrm{~km} \mathrm{~s}^{-1}$ (top three data points in Fig.6) and are clearly separated from the RV constant objects. Interestingly, the RV constant objects follow a clear trend of decreasing RV amplitude with increasing mass. On one hand, this reflects simply the depending of the RV precision on the signal-to-noise of the spectra. On the other hand, it is an interesting finding by itself, since it shows that this group of brown dwarfs and very low-mass stars with masses of $0.12 \mathrm{M}_{\odot}$ and below display no significant RV noise due to surface spots, which would cause systematic RV errors with a RV amplitude increasing with mass.

Among the subsample of ten brown dwarfs and very low-mass stars with masses $\leq 0.12 \mathrm{M}_{\odot}$ in this survey, only one $(\mathrm{Cha} \mathrm{H} \alpha 8)$ shows signs of RV variability, while the others are RV quiet with respect to both companions and spots in our observations. That hints at a very small multiplicity fraction of $10 \%$ or less. When considering also CHXR 74, i.e. eleven objects $\left(\mathrm{M} \leq 0.17 \mathrm{M}_{\odot}\right.$ and spectral types M4.5-M8), nine have constant RVs in the presented RV survey. Interestingly, Cha $\mathrm{H} \alpha 8$ is RV constant and CHXR 74 shows only small amplitude variations on time scales of days/weeks but both reveal larger amplitude RV variability only on longer time scales of at least several months. The fact that all other objects in this mass range do not show RV noise due to activity suggests that the sources for RV variability due to activity are also weak for Cha $\mathrm{H} \alpha$ 8. Furthermore, the timescales of the variability are much too long for being caused by rotational modulation since the rotational period 

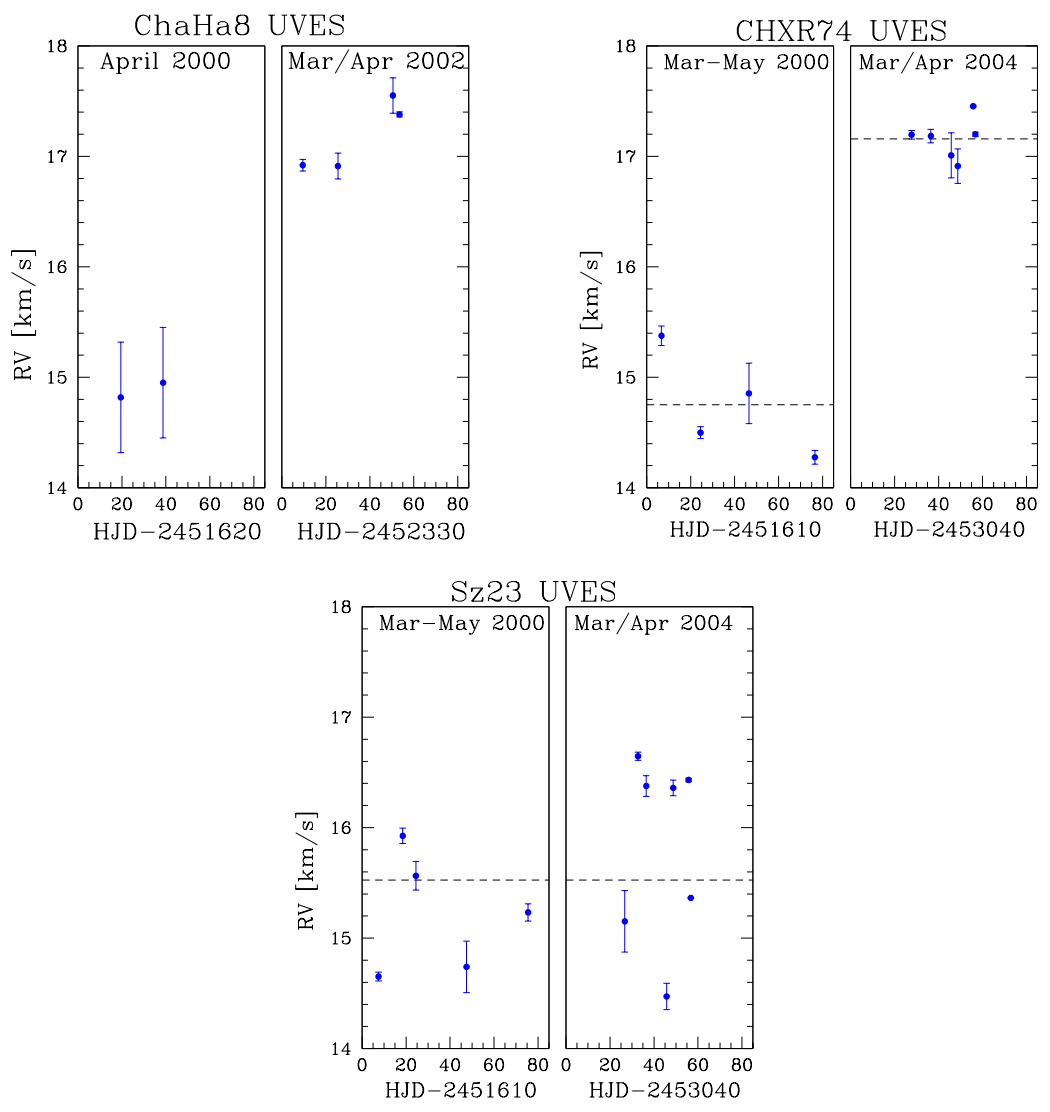

Figure 5: RV variable objects: RV vs. time in Julian days for the brown dwarf candidate Cha Ha 8 (M6.5) and the low-mass T Tauri stars CHXR 74 and Sz $23\left(\sim 0.2-0.3 \mathrm{M}_{\odot}\right)$ based on high-resolution UVES/VLT spectra. Error bars indicate $1 \sigma$ errors.

is of the order of 2 days. The only other explanation would be a companion with a mass of several Jupitermasses or more, i.e. a supergiant planet or a brown dwarf. These observations give hints that companions to young brown dwarfs and very low-mass stars might have periods of several months.

The RV survey probes the regions close to the central objects in respect of the occurrence of companions. Fig. $[7$ shows the separation ranges, which can be covered based on the achieved RV precision. For example, a 20 Jupitermass brown dwarf in orbit around a 60 Jupitermass brown dwarf would be detectable out to $10 \mathrm{AU}$ if the time base is long enough. For smaller companion masses the covered separation ranges are correspondingly smaller. At the current stage, the limits in the covered separation range is set by the time base rather than the RV precision. Therefore, further 3rd epoch RV measure- 


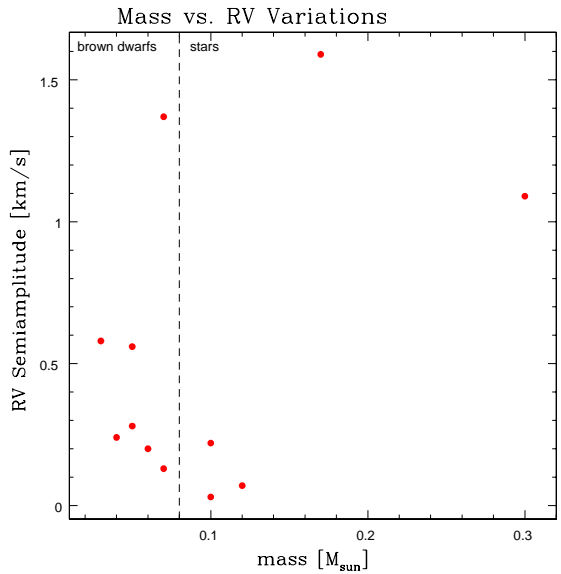

Figure 6: RV semiamplitude vs. object mass. The upper three data points represent the RV variable objects with amplitudes above $1 \mathrm{~km} \mathrm{~s}^{-1}$. The remaining data points represent RV constant objects, which clearly follow a trend of decreasing RV amplitude with increasing mass. indicating that they are displaying no significant RV noise due to surface spots down to the precision required to detect Jupitermass planets, which would cause and increasing RV amplitude with mass.

ments are planned. The found small multiplicity fraction of the brown dwarfs and very low-mass stars in Cha I at small separations in this RV survey, is also supported by the results of a direct imaging search for wide (planetary or brown dwarf) companions to mostly the same targets, Cha $\mathrm{H} \alpha 1-12$, by Neuhäuser et al. $(2002,2003)$, who find a multiplicity fraction of $\leq 10 \%$. The separation ranges covered by this HST survey are also indicated in Fig. 7 .

\section{Rotation}

Measurements of rotation rates of young brown dwarfs are important to determine the evolution of angular momentum in the substellar regime in the first several million years of their lifetime, during which rapid changes are expected due to the contraction on the Hayashi track, the onset of Deuterium burning and possible magnetic interaction with a circumstellar disk. Rotation speeds can be determined in terms of projected rotational velocities $v \sin i$ based on the line broadening of spectral features (Sect.6.1) or, if an object exhibits prominent surface features, which modulate the brightness as the object rotates, the absolute rotation period can be determined by a light curve analysis (Sect.6.2). Both techniques have been applied to the brown dwarfs and (very) low-mass stars in Cha I. 
RV survey and direct imaging: separation ranges
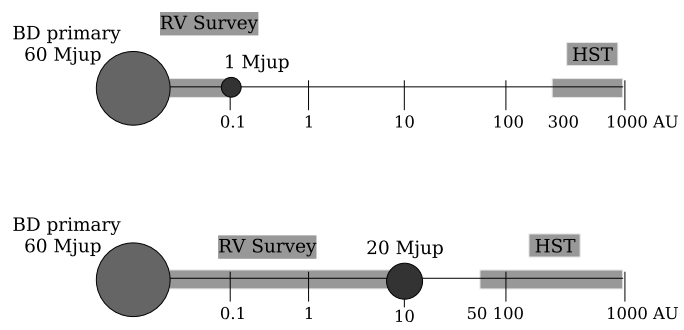

Figure 7: Separation ranges, which can be covered based on the achieved RV precision by our RV survey and by a direct imaging survey with the HST (Neuhäuser et al. 2002). An example is given for a one Jupitermass planet and a 20 Jupitermass brown dwarf orbiting a 60 Jupitermass brown dwarf. Due to limited time base not the whole possible range for the RV survey has been covered yet.

\subsection{Projected rotational velocities $v \sin i$}

Projected rotational velocities $v \sin i$ have been measured based on the line broadening of spectral lines in UVES spectra. We found that the $v \sin i$ values of the bona fide and candidate brown dwarfs in Cha I with spectral types M6-M8 range between $8 \mathrm{~km} \mathrm{~s}^{-1}$ and $26 \mathrm{~km} \mathrm{~s}^{-1}$. The spectroscopic rotational velocities of the (very) low-mass stars Cha $\mathrm{H} \alpha 4$, Cha $\mathrm{H} \alpha 5$, B 34, CHXR 74 and Sz 23 are 14-18 $\mathrm{km} \mathrm{s}^{-1}$ and, therefore, lie also within the range of that of the studied substellar objects. These measurements provided the first determination of projected rotational velocities for very young brown dwarfs (Joergens \& Guenther 2001). To compare them with $v \sin i$ values of older brown dwarfs, we consider that the late-M type brown dwarfs in Cha I at and age of 1-5 Myrs will further cool down and develop into L dwarfs at some point between and age of $100 \mathrm{Myr}$ and $1 \mathrm{Gyr}$ (Burrows et al. 2001) and later into $\mathrm{T}$ dwarfs. $v \sin i$ values for old $\mathrm{L}$ dwarfs range between 10 and $60 \mathrm{~km} \mathrm{~s}^{-1}$ with the vast majority rotating faster than $20 \mathrm{~km} \mathrm{~s}^{-1}$ (Mohanty \& Basri 2003). Thus, the brown dwarfs in Cha I rotate on average slower than old L dwarfs in terms of rotational velocities $v \sin i$. These results for brown dwarfs in Cha I are in agreement with $v \sin i$ values determined for five brown dwarf candidates in Taurus $\left(7-14 \mathrm{~km} \mathrm{~s}^{-1}\right.$, White \& Basri 2003) and for one brown dwarf in $\sigma$ Ori, which has a $v \sin i$ of $9.4 \mathrm{~km} \mathrm{~s}^{-1}$ (Muzerolle et al. 2003) and an absolute rotational velocity of $14 \pm 4 \mathrm{~km} \mathrm{~s}^{-1}$ (Caballero et al. 2004).

Projected rotational velocities $v \sin i$ are lower limits of the rotational 
velocity $v$ since the inclination $i$ of the rotation axis remains unknown. Based on $v \sin i$ and the radius of the object an upper limit of the rotational period $\mathrm{P} / \sin i$ can be derived. We estimated the radii of the brown dwarfs and very low-mass stars in Cha I by means of the Stefan-Boltzmann law from bolometric luminosities and effective temperatures given by Comerón et al. (1999, 2000). The approximate upper limits for their rotational periods range between one and three days for all studied brown dwarfs and (very) low-mass stars with the exception of the low-mass star CHXR $74\left(\mathrm{M} 4.5, \sim 0.17 \mathrm{M}_{\odot}\right)$, for which $\mathrm{P} / \sin i$ is five days.

\subsection{Absolute rotational periods from lightcurve modulations}

An object exhibiting prominent surface features distributed inhomogeneously over its photosphere provides a way to measure its absolute rotation period since the surface spot(s) cause a periodic modulation of the brightness of the object as it rotates. See Fig. [8 for an illustration.

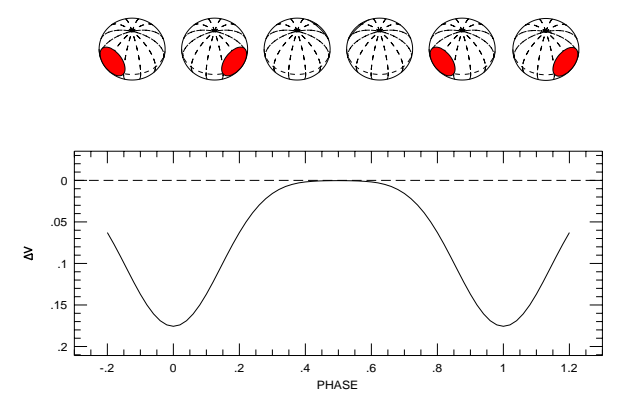

Figure 8: Rotational brightness modulation caused by surface spot. Courtesy of G. Torres. A cool spot on the surface of a star / brown dwarf causes a periodic dimming of the total brightness at the rotation period. Plotted are the relative $\mathrm{V}$ magnitudes $\Delta \mathrm{V}$ over the orbital phase.

Based on photometric observations in the $\mathrm{R}$ and i band filter (see Sect.3.3), we searched for periodic variations in the light curves of the targets with the string-length method (Dworetsky 1983). Periodic brightness variations were found for the three brown dwarf candidates Cha $\mathrm{H} \alpha 2$, Cha $\mathrm{H} \alpha 3$, Cha $\mathrm{H} \alpha 6$ and two very low-mass stars B 34 and CHXR 78C. The original light curves are shown in Fig. 9. In addition to i and $\mathrm{R}$ band data, we have analysed J-band monitoring data of the targets (Carpenter et al. 2002), which have been taken a few weeks earlier and confirm the periods found in the optical data. The determined periods are interpreted as rotation periods based on a consistency with the $v \sin i$ values from UVES spectra (previous section), recorded color variations in agreement with the expectations for spots on the surface and the fact that with effective temperatures of more than $2800 \mathrm{~K}$, the objects 

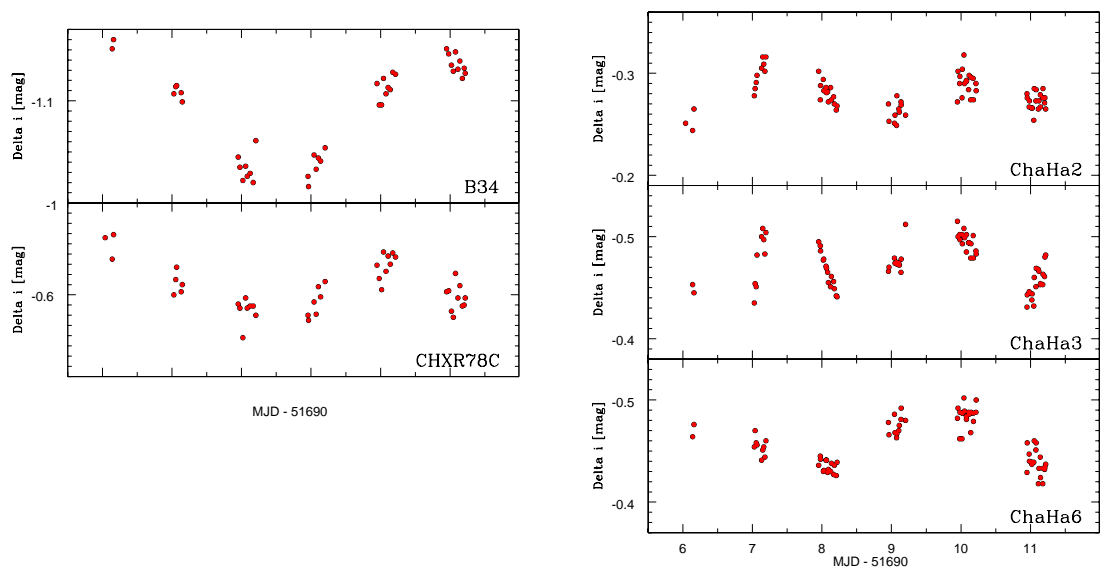

Figure 9: Light curves for two very low-mass stars (left panel) and three brown dwarf candidates (right panel). From top to bottom and left to right are displayed i-band light curves for the very low-mass stars B34 (M5, $0.12 \mathrm{M}_{\odot}$ ) with a period of 4.5 days and CHXR78C (M5.5, $\left.0.09 \mathrm{M}_{\odot}\right)$ with a period of 3.9 days, and for the three brown dwarf candidates $\mathrm{ChaH} \alpha 2\left(\mathrm{M} 6.5,0.07 \mathrm{M}_{\odot}\right)$ with a period of 2.8 days, Cha $\mathrm{H} \alpha 3\left(\mathrm{M} 7,0.06 \mathrm{M}_{\odot}\right)$ with a period of 2.2 days and $\mathrm{Cha} \mathrm{H} \alpha 6(\mathrm{M} 7$, $\left.0.05 \mathrm{M}_{\odot}\right)$ with a period of 3.4 days.

are young and still hot and, therefore, their atmospheric gas is very likely sufficiently ionized for the formation of spots. Additionally, variability due to clouds, which could occur on time scales of the formation and evolution of clouds, can be excluded because their temperatures are too high for significant dust condensation (e.g. Tsuji et al. 1996a,b, Allard et al. 1997, Burrows \& Sharp 1999).

The found rotational periods of the three brown dwarf candidates Cha $\mathrm{H} \alpha 2$, Cha $\mathrm{H} \alpha 3$ and Cha $\mathrm{H} \alpha 6$ are $3.2 \mathrm{~d}, 2.2 \mathrm{~d}$ and $3.4 \mathrm{~d}$, respectively (Joergens et al. $2003 \mathrm{~b}$ ). The results show that brown dwarfs at an age of $1-5$ Myr display an inhomogeneous surface structure and rotate slower than old brown dwarfs (rotational periods below one day, e.g. Bailer-Jones \& Mundt 2001, Martín et al. 2001). It is known that $\mathrm{ChaH} \alpha 2$ and 6 have optically thick disks (Comerón et al. 2000), therefore magnetic braking due to interactions with the disk may play a role for them. This is suggested by the fact, that among the three brown dwarf candidates with determined periods, the one without a detected disk, Cha $\mathrm{H} \alpha 3$, has the shortest period.

\subsection{Rotation of young brown dwarfs}

The brown dwarfs in Cha I have rotation periods of $2-3$ days and projected rotation velocities $v \sin i$ of $8-26 \mathrm{~km} \mathrm{~s}^{-1}$ as shown by our observations. Their rotation periods are significantly larger than those for old brown dwarfs (below 
one day, e.g. Bailer-Jones \& Mundt 2001) and their rotational velocities are on average smaller than for old brown dwarfs $\left(10-60 \mathrm{~km} \mathrm{~s}^{-1}\right.$, Mohanty \& Basri 2003). This is in agreement with the idea that they are in an early contracting stage and will further spin up and contract.

Periodic photometric variabilities have been also detected for two dozen substellar $\sigma$ Ori and $\epsilon$ Ori members and member candidates (Bailer-Jones \& Mundt 2001, Zapatero Osorio et al. 2003, Scholz \& Eislöffel 2004a,b, Caballero et al. 2004). Including our periods in Cha I, the to-date known photometric periods for young brown dwarfs cover a wide range from 46 min to 3.4 days. Apart from rotational modulation due to chromospheric spots, several other possible processes have been suggested to account for the periodic variabilities, like accretion phenomena, formation and evolution of dust coverage for the cooler ones and eclipsing companions, which could all occur on various time scales. Among all known periods for young brown dwarfs, only four have been confirmed as rotational periods by spectroscopic measurements of their rotational velocities $v \sin i$ (Cha $\mathrm{H} \alpha 2$, Cha $\mathrm{H} \alpha 3$, Cha $\mathrm{H} \alpha 6$, S Ori 25, Joergens \& Guenther 2001, Joergens et al. 2003b, Muzerolle et al. 2003, Caballero et al. 2004), they all lie between 1.7 and 3.5 days. Several periods of the order of a few hours have been also interpreted as rotational periods. However, as noticed by Joergens et al. (2003b), rotation periods of a few hours for very young brown dwarfs imply extreme rotation with rotational velocities of $100 \mathrm{~km} \mathrm{~s}^{-1}$ or more given the still large radii at this young age. These speeds come close to break-up velocities and for the $46 \mathrm{~min}$ period, Zapatero Osorio et al. (2003) found that it is indeed above break-up velocity and cannot be a rotation period. The finding of a rotation of $100 \mathrm{~km} \mathrm{~s}^{-1}$ or more for brown dwarfs at a few million years would also be surprising in respect of the fact that they are in an early contracting stage and the expectation of a further spin-up in their future evolution. A check of the proposed extreme rapid rotation by $v \sin i$ measurements would be desirable. So far, all $v \sin i$ determinations for young brown dwarfs in Cha I, Taurus and $\sigma$ Ori indicate rotational velocities below $26 \mathrm{~km} \mathrm{~s}^{-1}$ with the vast majority below $20 \mathrm{~km} \mathrm{~s}^{-1}$ (Joergens \& Guenther 2001, White \& Basri 2003, Muzzerolle et al. 2003).

\section{Summary and conclusions}

The presented work reports about observations of a population of very young brown dwarfs and low-mass stars close to the substellar borderline in the Cha I star forming region. At an age of only a few million years, their exploration allows insights into the formation and early evolution of brown dwarfs. The targets were studied in terms of their kinematic properties, the occurrence of multiple systems among them as well as their rotational characteristics.

We are carrying out a RV survey for planets and brown dwarf companions to the targets with UVES at the VLT. The achieved RV precision allows us to search for companions down to Jupiter mass planets. The analysis of the high-resolution spectra reveals very constant RVs on time scales of weeks to 
months for the majority of the targets as well as RV variability for three sources (Joergens et al. 2005a). The RV constant objects are six brown dwarfs and three very low-mass stars $\left(\mathrm{M} \leq 0.12 \mathrm{M}_{\odot}\right.$, spectral types $\left.\mathrm{M} 5-\mathrm{M} 8\right)$, for which we estimate upper limits for masses of hypothetical companions to lie between $0.1 \mathrm{M}_{\mathrm{Jup}}$ and $1.5 \mathrm{M}_{\mathrm{Jup}}$. This group shows a relation of decreasing RV amplitude with increasing mass. This reflects simply a higher RV precision for more massive targets due to a better signal-to-noise, whereas the effect of RV errors caused by surface features would be the opposite. This demonstrates that brown dwarfs and very low-mass stars $\left(\mathrm{M} \leq 0.12 \mathrm{M}_{\odot}\right)$ in Cha I display no significant RV noise due to surface spots down to the precision necessary to detect Jupitermass planets. Thus, they are suitable targets to search for planets with the RV technique.

Three objects exhibit significant RV variations with peak-to-peak amplitudes of $2-3 \mathrm{~km} \mathrm{~s}^{-1}$ : the brown dwarf Cha $\mathrm{H} \alpha 8$ and the low-mass stars CHXR $74\left(\sim 0.17 \mathrm{M}_{\odot}\right)$ and Sz $23\left(\sim 0.3 \mathrm{M}_{\odot}\right)$. For Sz 23 , which is the highest mass object in our sample, we have indications that the RV variations are caused by surface spots from the variability time scale and from significant CaII IR triplet emission. Cha $\mathrm{H} \alpha 8$ and CHXR 74 show a different variability behaviour with displaying only very small amplitude or no variations on time scales of days to weeks but significant RV variations on times scales of months or longer, which cannot be explained by being rotational modulation. If caused by orbiting companions, the detected RV variations of CHXR 74 and $\mathrm{Cha \textrm {H }} \alpha 8$ correspond to giant planets of a few Jupiter masses with periods of several months. In order to explore the nature of the detected RV variations follow-up observations of CHXR 74 and $\mathrm{Cha} \mathrm{H} \alpha 8$ are planned. If confirmed as planetary systems, they would be unique because they would contain not only the lowest mass primaries and the first brown dwarf with a planet but with an age of a few million years also the by far youngest extrasolar planets found to date. That would provide empirical constraints for planet formation time scales as well as for the formation of brown dwarfs.

The found multiplicity fraction in this survey is obviously very small. Considering the subsample of the ten brown dwarfs and very low-mass stars with masses $\leq 0.12 \mathrm{M}_{\odot}$, only one of them (Cha $\left.\mathrm{H} \alpha 8\right)$ shows signs of RV variability, while the others are RV quiet with respect to both companions and spots in our observations. That hints at a very small multiplicity fraction of $10 \%$ or less. However, the RV variable brown dwarf $\mathrm{Cha \textrm {H }} \alpha 8$ and also the higher mass CHXR 74 (not included in the above considered subsample) are hinting at the possibility that companions to young brown dwarfs and very low-mass stars have periods of several months and such a time scale was not covered for all targets. Thus, our results of small multiplicity reflects so far mainly separations of around $0.1 \mathrm{AU}$. Further 3rd epoch RV determinations are planned. At much larger separations, a direct imaging search for wide (planetary or brown dwarf) companions to mostly the same targets also found a very small multiplicity fraction of $\leq 10 \%$ (Neuhäuser et al. 2002, 2003). There still remains a significant gap in the studied separation ranges, which will partly be probed by the planned follow-up 3rd epoch RV measurements and partly 
is only accessible with high-resolving AO imaging (NACO / VLT) or even requires interferometric techniques (e.g. AMBER at the VLTI). For the already studied separations, the overall picture is a multiplicity fraction significantly smaller than for $\mathrm{T}$ Tauri stars even when taking into account the smaller available mass range for the companions. This hints at differences in the formation processes of brown dwarfs and $\mathrm{T}$ Tauri stars.

In order to test the proposed ejection scenario for the formation of brown dwarfs, we explored the kinematic properties of our substellar targets based on absolute mean RVs derived within the presented RV survey. We find that the brown dwarfs in Cha I form also kinematically a very homogeneous group. They have very similar absolute RVs with a RV dispersion in terms of standard deviation of only $0.9 \mathrm{~km} \mathrm{~s}^{-1}$ (Joergens \& Guenther 2001, Joergens et al. $2005 \mathrm{~b}$ ). A study of T Tauri stars in the same field showed that there are no indications for a more violent dynamical evolution, like more frequent ejections, for the brown dwarfs compared to the T Tauri stars since the RV dispersion of the $\mathrm{T}$ Tauri stars $\left(1.3 \mathrm{~km} \mathrm{~s}^{-1}\right)$ was determined to be even slightly larger than that for the brown dwarfs. This is the first observational constraint for the velocity distribution of a homogeneous group of closely confined very young brown dwarfs and therefore a first empirical upper limit for ejection velocities.

Theoretical models of the ejection scenario have been performed by several groups in recent years. Some of them are hinting at the possibility of a only small or of no mass dependence of the velocities. Thus, the fact that we do not find a larger velocity dispersion for the brown dwarfs than for the $\mathrm{T}$ Tauri stars does not exclude the ejection scenario. However, we observe smaller velocities than any of the theoretical predictions for brown dwarfs formed by the ejection scenario. This might be partly attributed to the fact that our sample is statistically relatively small, or it might be explained by the lower densities in Cha I compared to some model assumptions, or by shortcomings in the models, like neglection of feedback processes (Bate et al. 2003, Bate \& Bonnell 2005, Delgado-Donate et al. 2004) or of the cluster potential (Sterzik \& Durisen 2003, Umbreit et al. 2005). The current conclusion is that either the brown dwarfs in ChaI have been formed by ejection but with smaller velocities as theoretically predicted or they have not been formed in that way. We are planning to enlarge the sample in the future to put the results on an improved statistical basis.

Finally, we studied the rotational properties of the targets in terms of projected rotational velocities $v \sin i$ measured in UVES spectra as well as in terms of absolute rotational periods derived from light curve analysis. We found that the $v \sin i$ values of the bona fide and candidate brown dwarfs in Cha I range between $8 \mathrm{~km} \mathrm{~s}^{-1}$ and $26 \mathrm{~km} \mathrm{~s}^{-1}$. These were the first determinations of rotational velocities for very young brown dwarfs (Joergens \& Guenther 2001). Furthermore, we have determined rotational periods, consistent with $v \sin i$ values, by tracing modulations of the light curves due to surface spots at the rotation period. We found periods for the three brown dwarf candidates Cha $\mathrm{H} \alpha 2$, Cha $\mathrm{H} \alpha 3$ and $\mathrm{Cha} \mathrm{H} \alpha 6$ of 2-3 days and for two very low-mass stars B 34 and CHXR78C $\left(\mathrm{M} \leq 0.12 \mathrm{M}_{\odot}\right)$ of $4-5$ days (Joergens 
et al. 2003b). Magnetic braking due to interactions with a circum-stellar disk may play a role for some of them since the ones with detected disks are the slower rotators.

The emerging picture of the rotation of young brown dwarfs at an age of a few million years based on rotational velocities for brown dwarfs in Cha I, Taurus and $\sigma$ Ori (Joergens \& Guenther 2001, White \& Basri 2003, Muzzerolle et al. 2003) and on spectroscopically confirmed rotation periods (our three brown dwarfs in Cha I plus one $\sigma$ Ori brown dwarf, Caballero et al. 2004) indicates that young brown dwarfs rotate with periods of the order of a few days and speeds of 7 to $26 \mathrm{~km} \mathrm{~s}^{-1}$. Their rotation periods are significantly larger than those for old brown dwarfs (below one day, e.g. Bailer-Jones \& Mundt 2001) and their rotational velocities are on average smaller than for old brown dwarfs (10-60 $\mathrm{km} \mathrm{s}^{-1}$, e.g. Mohanty \& Basri 2003). This is in agreement with the idea that they are in an early contracting stage and will further spin up and contract before they reach a final radius when their interior electrons are completely degenerate.

Despite the fact that the origins of brown dwarfs are still shrouded in mist, we think that the presented comprehensive observations of very young brown dwarfs in Cha I and the determination of their fundamental parameters brought us an important step forward in revealing the details of one of the main open issues in stellar astronomy and in the origins of solar systems.

\section{Acknowledgements}

It is a pleasure to acknowledge fruitful collaborations in the last years on the subject of this article with Ralph Neuhäuser, Eike Guenther, Matilde Fernández, Fernando Comerón and Günther Wuchterl. Furthermore, I am grateful for a grant from the 'Deutsche Forschungsgemeinschaft' (Schwerpunktprogramm 'Physics of star formation') during my $\mathrm{PhD}$ time as well as current support by the European Union through a Marie Curie Fellowship under contract number FP6-501875.

\section{References}

Allard F., Hauschildt P.H., Alexander D.R., Starrfield S. 1997 ARA\&A 35, 137

Armitage, P. J.; Clarke, C. J. 1997, MNRAS, 285, 540

Bailer-Jones C.A.L., Mundt R. 2001, A\&A 367, 218

Basri G., Martín E.L. 1999, ApJ 118, 2460

Basri G. 2000, ARA\&A 38, 485

Bate M.R., Bonnell I.A., Bromm V. 2003, MNRAS 339, 577

Bate M.R., Bonnell I.A. 2005, MNRAS 356, 1201

Béjar V.J.S., Zapatero Osorio M.R., Rebolo R. 1999, ApJ 521, 671

Burrows A., Sharp C.M. 1999, ApJ 512, 843

Burrows A., Hubbard W.B., Lunine J.I., Liebert J. 2001, Rev. Mod. Phys. 73, 3 
Caballero J.A., Béjar V.J.S., Rebolo R., Zapatero Osorio M.R. 2004, A\&A 424, 857

Carpenter J.M., Hillenbrand L.A., Skutskie M.F., Meyer M.R. 2002, AJ 124, 1001

Chauvin G., Lagrange A.-M., Dumas C. et al. 2004, A\&A 425, L29

Comerón F., Rieke G.H., Neuhäuser R. 1999, A\&A 343, 477

Comerón F., Neuhäuser R., Kaas A.A. 2000, A\&A 359, 269

Covino E., Alcalá J.M, Allain S. et al. 1997, A\&A 328, 187

Dekker H., D’Odorico S., Kaufer A., Delabre B., Kotzlowski H. 2000, In: SPIE Vol. 4008, p. 534, ed. by M.Iye, A.Moorwood

Delfosse X., Tinney C.G., Forveille T. et al. 1997, A\&A 327, L25

Delgado-Donate E.J., Clarke C.J., Bate M.R. 2003, MNRAS 342, 926

Delgado-Donate E.J., Clarke C.J., Bate M.R. 2004, MNRAS 347, 759

Dubath P., Reipurth B., Mayor M. 1996, A\&A 308, 107

Durisen R.H., Sterzik M.F., Pickett B.K. 2001, A\&A 371, 952

Dworetsky M.M. 1983, MNRAS 203, 917

Ghez A.M., Neugebauer G., Matthews K. 1993, AJ 106, 2005

Guenther E.W., Wuchterl G. 2003, A\&A 401, 677

Hawley S.L., Covey K.R., Knapp G.R. et al. 2002, AJ 123, 3409

Joergens V., Guenther E. 2001, A\&A 379, L9

Joergens V. 2003, PhD thesis, Ludwigs-Maximilians Universität München

Joergens V., Fernández M., Carpenter J.M., Neuhäuser R. 2003b, ApJ 594, 971

Joergens V., Neuhäuser R., Guenther E.W., Fernández M., Comerón F., In: IAU Symposium No. 211, Brown Dwarfs, ed. by E. L. Martín, Astronomical Society of the Pacific, San Francisco, 2003a, p.233

Joergens V. et al. 2005a, in prep.

Joergens V. et al. 2005b, in prep.

Kirkpatrick J.D., Reid I.N., Liebert J. et al. 2000, AJ 120, 447

Köhler R., Kunkel M., Leinert Ch., Zinnecker H. 2000, A\&A 356, 541

Kroupa, P., Bouvier, J. 2003, MNRAS 346, 369

Kumar S. 1962, AJ 67, 579

Kumar S. 1963, ApJ 137, 1121

Leinert Ch., Zinnecker H., Weitzel N. et al. 1993, A\&A 278, 129

Low C., Lynden-Bell D. 1976, MNRAS 176, 367

Luhman K. 2004, ApJ 602, 816

Martín E.L., Brandner W., Bouvier J. et al. 2000 ApJ 543, 299

Martín, E. L., Zapatero Osorio M. R. \& Lehto H.J. 2001, ApJ, 557, 822

Mayor M., Queloz D. 1995, Nature 378, 355

Mayor, M., Udry, S., Naef, D. et al. 2003, A\&A 415, 391 
Mizuno A., Hayakawa T., Tachihara K. et al. 1999, PASJ 51, 859

Mohanty S., Basri G. 2003, ApJ 583, 451

Muzerolle J., Hillenbrand L., Calvet N., Briceño C., Hartmann L. 2003, ApJ, 592, 266

Nakajima T., Oppenheimer B.R., Kulkarni S.R. et al. 1995, Nature 378, 463

Neuhäuser R., Comerón F. 1998, Science 282, 83

Neuhäuser R., Comerón F. 1999, A\&A 350, 612

Neuhäuser R., Brandner W., Alves J., Joergens V., Comerón F. 2002, A\&A 384, 999

Neuhäuser R., Brandner W., Guenther E. 2003, IAUS 211, 309, ed. by E. Martín

Oppenheimer B.R., Kulkarni S.R., Matthews K., Nakajima T. 1995 Science 270, 1478

Rebolo R., Martín E.L., Magazzú A. 1992, ApJ 389, L83

Rebolo R., Zapatero Osorio M.R., Martín E.L. 1995, Nature 377, 129

Reipurth B., Clarke C. 2001, ApJ 122, 432

Santos N.C., Bouchy F., Mayor M., Pepe F., Queloz D. et al. 2004, A\&A 426, L19

Scholz A., Eislöffel J. 2004a, A\&A 419, 249

Scholz A., Eislöffel J. 2004b, A\&A in press, astro-ph/0410101

Shu F.H., Adams F.C., Lizano S. 1987, ARA\&A 25, 23

Stauffer J.R., Hamilton D., Probst R. 1994, AJ 108, 155

Sterzik M.F., Durisen R.H. 1995, A\&A 304, L9

Sterzik M.F., Durisen R.H. 1998, A\&A 339, 95

Sterzik M.F., Durisen R.H. 2003, A\&A, 400, 1031

Tsuji T., Ohnaka K., Aoki W. 1996a, A\&A 305, L1

Tsuji T., Ohnaka K., Aoki W., Nakajima T. 1996b, A\&A 308, L29

Umbreit S., Burkert A., Henning T., Mikkola S., Spurzem R. 2005, A\&A, in press

Valtonen M., Mikkola S. 1991, ARA\&A 29, 9

Walter F.M. 1992, AJ 104, 758

White, R. J. \& Basri, G. 2003, ApJ 582, 1109

Whitworth, A.P., Zinnecker, H. 2004, A\&A 427, 299

Wuchterl G., Guillot T., Lissauer J.J. 2000. In: Protostars and Planets IV, ed. by Mannings V., Boss A.P., Russell S.S., Univ. of Arizona Press, Tucson, p.1081

Zapatero Osorio M.R., Caballero J.A., Béjar V.J.S., Rebolo R. 2003, A\&A 408, 663 